\title{
Comparison with Surgical Findings for the Accuracy of Routine MRI in Rotator Cuff Tears
}

\author{
Narendra Darai 1,2, Suvash Pokhrel3, Rongbao Shu4, Xiaojuan Zhang5, Jiacheng Liu1,2, \\ Gaojun Teng1,2* \\ ${ }^{1}$ Department of Radiology, Zhong Da Hospital, Southeast University, Nanjing, China \\ ${ }^{2}$ Medical School, Southeast University, Nanjing, China \\ ${ }^{3}$ Department of Internal Medicine, Xinxiang Medical University, Xinxiang, China \\ ${ }^{4}$ Department of Radiology, People's Hospital of Maanshan, Maanshan, China \\ ${ }^{5}$ Department of Public Health, Zhengzhou Medical University, Zhengzhou, China \\ Email: darainaren@hotmail.com,dr.suvashpokhrel@gmail.com, shurongb@qq.com, truezxj@163.com, \\ jcheng_liu@163.com, "gjteng@vip.sina.com
}

Received 17 March 2016; accepted 30 May 2016; published 2 June 2016

Copyright (C) 2016 by authors and Scientific Research Publishing Inc.

This work is licensed under the Creative Commons Attribution International License (CC BY).

http://creativecommons.org/licenses/by/4.0/

c) (i) Open Access

\begin{abstract}
Objective: To evaluate the diagnostic efficacy of magnetic resonance imaging (MRI) for the detection of partial-thickness rotator cuff tears (PTT) and full-thickness rotator cuff tears(FTT) by comparing its findings with surgical findings as the gold standard and to improve the previous MRI accuracy in diagnosing rotator cuff tears (RCT) considering more variables. Methods: In 45 months, 804 patients underwent MRI shoulder joint. Among them, only 95 cases had undergone both MRI imaging and surgery accordingly. The patient records were evaluated retrospectively if MRI and surgery were performed within 40 days of MRI. MRI findings were categorized into PTT, FTT and no tears which were further divided into different types according to four main nominal data as variables viz. site, size, shape and muscle involvement in RCT and were correlated with surgical findings for statistical calculation by using Kappa coefficient and McNemar Bowker test. Results: 81 patients ( 86 RCTs) underwent surgery within 40 days. On the basis of site as variable, MRI correctly depicted $100 \%$ of full thickness tears(FTT), $85 \%$ of bursal partial thickness tears(PTT), 80.4\% of articular partial thickness tears(PTT). The consistency in diagnosis of RCT between MRI and surgery was moderate (Kappa coefficient 0.645 ). Overall sensitivity, specificity and accuracy of MRI for diagnosing PTT was $87.3 \%, 53.3 \%$ and $81.3 \%$; and that for FTT was $100 \%$, $\mathbf{9 8 . 7 \%}$ and $98.8 \%$ respectively. Likewise on the basis of size, shape and muscles involved, the consistency between MRI and surgery was poor for size and shape and moderate for muscles involved; and the difference in diagnosing RCT by MRI and surgery was significant for shape $(P=0.002)$ only,
\end{abstract}

${ }^{*}$ Corresponding author.

How to cite this paper: Darai, N., Pokhrel, S., Shu, R.B., Zhang, X.J., Liu, J.C. and Teng, G.J. (2016) Comparison with Surgical Findings for the Accuracy of Routine MRI in Rotator Cuff Tears. Open Journal of Radiology, 6, 73-83.

http://dx.doi.org/10.4236/ojrad.2016.62011 
but not significant for size $(P=0.16)$ and for muscles involved $(P=0.206)$ respectively. The agreement between MRI and surgery in diagnosing calcific tendinitis and shoulder joint hematoma with Kappa coefficient is $(0.577)$ and $(0.556)$ respectively. Conclusion: MRI has better accuracy for detecting FTT and has high sensitivity and positive predictive value in diagnosing both PTT and FTT. Combining more others variables in addition to RCT, MRI offers a great value in diagnosing RCT.

\section{Keywords}

\section{Shoulder Joint, Partial-Thickness Rotator Cuff Tears (PTT), Full-Thickness Rotator Cuff Tears (FTT), Magnetic Resonance Imaging (MRI)}

\section{Introduction}

Rotator cuff tears (RCT) are one of the most common causes of shoulder pain for which patients seek treatment. As in our daily work, the shoulder joint is the most frequently used, there is higher chance of having shoulder joint injury. Thirty to seventy percent of patients having shoulder pain are due to abnormality of rotator cuff [1] [2]. The decision making in management of causes of shoulder pain depends upon the results of imaging of the shoulder. The accurate diagnosis of rotator cuff tears by magnetic resonance imaging (MRI) is a must for an orthopedic surgeon to proceed with surgery [3]. MRI became the favored investigation for preoperative diagnosis of partial- and full-thickness rotator cuff tears as it can confirm a suspected RCT non-invasively [4]-[6]. It can reveal the RCT for necessary surgical procedures although its accuracy greatly depends on the skill of the reporters in interpreting a MRI slides. According to the literature regarding accuracy of MRI is still controversial in the diagnosis of rotator cuff tears despite less invasive approach. On the basis of availability, clinical experience and the expected sensitivity and specificity, accuracy of MRI can be assessed [7].

Many literatures have shown the accuracy of MRI for the detection of partial-thickness rotator cuff tears (PTT) and full-thickness rotator cuff tears (FTT) [8] [9], but most of them have relatively small number of patient with field strength of MRI less than $3.0 \mathrm{~T}$ and have considered only few parameters [10]-[12]. Dinnes et al.; a systemic review of diagnostic accuracy of MRI in the detection of rotator cuff tears; showed that MRI have high accuracy for the detection of FTT in comparison to PTT [13]. According to the available information, MRI is the first choice as the diagnostic investigation for RCT in our hospital.

The objective of this study is to evaluate the diagnostic efficacy of MRI in RCT and compare the accuracy of MRI for the detection of PTT and FTT using surgical findings as the "gold standard" and to improve the previous MRI accuracy for diagnosing RCT considering more variables.

\section{Materials and Methods}

In this retrospective study, the inclusion criteria were: (1) shoulder discomfort and/or disability for which the patients underwent MRI; (2) also subsequent surgery was carried out; (3) no history of previous shoulder surgery; (4) healthy contralateral shoulder; (5) informed consent for surgery. The indications to perform MRI were clinical suspicion for having injury of rotator cuff muscles and intra- and extra-articular structures of the shoulder joint that are responsible for restriction of normal shoulder movement $(n=45)$, as a request for more diagnostic certainty who were previously suspected in another center as rotator cuff injury $(n=19)$, the patients who didn't improve by medical and conservative management $(n=11)$ and of the remaining patients $(n=6)$, the indication to do MRI could not be assessed.

Patients were excluded when case was refused for surgery, also when the patients who underwent surgery but not arthroscopy or open surgery, when MRI images were of bad quality and when the time intervals between MRI and surgery exceeded 2 months and when RCT was absent in both MRI and surgery i.e. when true negative cases were present.

The study was performed in a tertiary teaching hospital under the supervision of an assistant professor and a professor. The cases were referred by the orthopedic department or general practitioners from January 1, 2012 to September 30, 2015. Total number of cases of upper limb imaging was 1474 between that time interval, out of 
them 804 cases had involved the shoulder joint. Among them, 95 cases ( 37 male and 58 female) underwent MRI of the affected shoulder joint and all of them subsequently had surgery. 12 cases; although managed surgically after the MRI diagnosis of RCT; were excluded as they were not undergone arthroscopic or open surgery. Also 2 more cases were excluded because the time period between MRI and surgery exceeded 2 months. The remaining 81 cases (30 males and 51 females, with a mean age of 56 years, range 19 - 83 years) formed the study group whose data obtained from MRI and surgical reports were analyzed retrospectively considering the surgical findings as the "gold standard". Out of 81 cases, 86 RCTs were found (2 RCTs for each 5 cases and 1 RCT for each 76 cases).

All those cases of the study group were searched thoroughly for the presence or absence of rotator cuff tears. All the findings were categorized into partial thickness tears (PTT), full thickness tears (FTT) and no tears. Further those tears had been divided into different types: A. according to the site as articular partial tear, bursal partial tear, intra-tendinous partial tear, full thickness tear and no tears; B. according to the size as less than $1 \mathrm{~cm}, 0$ to less than $2 \mathrm{~cm}, 2 \mathrm{~cm}$ to less than $3 \mathrm{~cm}, 3 \mathrm{~cm}$ or greater than $3 \mathrm{~cm}$ and no tears; C. according to the shape as crescentic, L-shape, U-shape and no tears; D. according to the muscle involved as supraspinatus, infraspinatus, teres minor, subscapularis and no tears; and the associated findings were recorded as absent or present for evaluation. The time interval between the MRI and surgery ranges from 1 to 40 days.

\section{Magnetic Resonance Imaging (MRI)}

All MRI examinations were performed with 3.0 Tesla (T) MR system (brand: Siemens, Germany). All the patients underwent routine MRI. The routine MRI of shoulder consisted of coronal T2 weighted imaging (T2WI) with fat-supression (FS) and T1 weighted image (T1WI) turbo spin echo, sagittal T2WI with fat-supression (FS) and axial T2WI with fat-suppression (FS). A field view of $200 \mathrm{~mm}$ was used, the slice thickness was $3 \mathrm{~mm}$, the imaging matrix was $320 \mathrm{~mm} \times 320 \mathrm{~mm}$ and overall imaging time was 4 minutes.

The MRI findings were reported by 3 experienced radiologists and these findings were retrospectively evaluated to compare with surgical findings. The cases; which were reported by experienced radiologists who have good enough anatomical, pathological and diagnostic radiological knowledge of shoulder joint; were chosen to exclude the potential bias of correct findings.

Recognized classification were used for the diagnosis of types of RCT and to categorize them for statistical calculation [4] [14]-[16]. Two MRI images have been shown to show partial thickness tear (PTT) and full thickness tear (FTT) in Figure 1 and Figure 2.

\section{Surgery}

The surgery either arthroscopy or open surgery was done by an experienced orthopedic surgeon having good

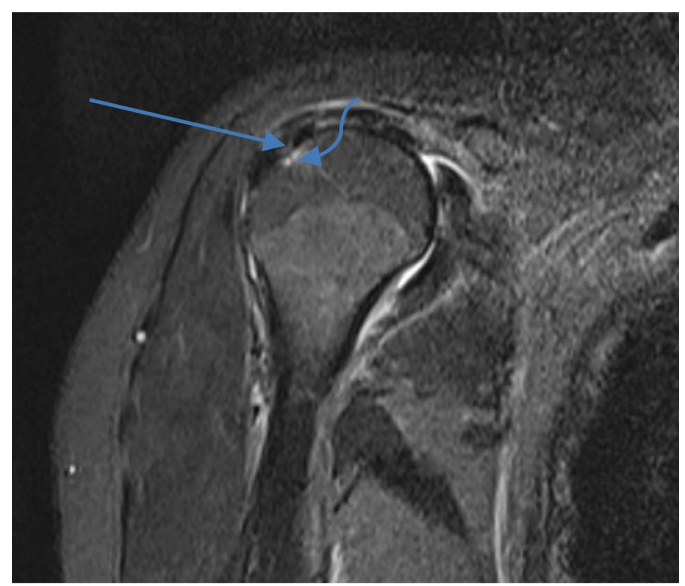

Figure 1. Coronal T2 weighted fat-suppression: MRI appearance of partial thickness tear (arrow) at the insertion of supraspinatus tendon. Also humeral head edema (curved arrow) and shoulder joint effusion can be seen. 


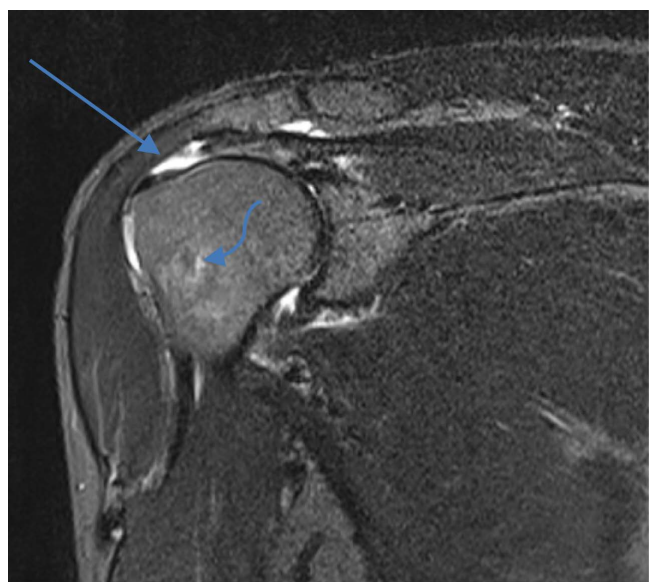

Figure 2. Coronal T2 weighted fat-suppression: MRI appearance of full thickness tear (arrow) at the insertion of supraspinatus tendon. Also humeral head edema (curved arrow) and shoulder joint effusion can be seen.

enough knowledge of the MRI findings. In all cases, the presence or absence of a partial-thickness or fullthickness rotator cuff tears and all associated other intra- as well as extra-articular lesions that are mentioned in surgical reports were recorded.

\section{Statistical Analysis}

The presence or absence of rotator cuff tears according to their site, size, shape and muscles involved were compared between MRI and surgery using cross tabulations. Also the presence or absence of other extra- or intraarticular lesions was compared as individual variables to find out their individual statistical accuracy. Sensitivity, specificity, the positive predictive value and the negative predictive value were calculated based on the tables.

A Kappa coefficient was calculated to show the agreement between the results of MRI and surgery. The statistical significance were determined in the differences of detecting rotator cuff tears by using marginal homogeneity test (or McNemar test) accordingly. Using IBM SPSS statistics Version 21.0, all the data were analyzed.

\section{Results}

At surgery 73 were partial-thickness rotator cuff tears (PTT), 7 were full-thickness rotator cuff tears (FTT) and 6 were no tear. On evaluating rotator cuff tears on the basis of site as a variable, MRI correctly identified 67 (83.75\%) out of total 80: 7 (100\%) of 7 FTT, 23 (85.1\%) of 27 bursal PTT, 37 (80.4\%) of 46 articular PTT (Table 1).

Overall sensitivity and specificity of MRI in diagnosing partial-thickness rotator cuff tears was 62/71 (87.3\%) and 8/15 (53.3\%); and that for diagnosing full-thickness rotator cuff tears was 7/7 (100\%) and 78/79 (98.7\%) respectively. Table 2 shows the sensitivity, specificity, positive predictive value, negative predictive value and accuracy for MRI in the detection of partial-thickness and full-thickness rotator cuff tears.

The agreement between MRI and surgery was moderate with general consistency: Kappa coefficient was calculated to be 0.645 (Table 3). The differences in the diagnosis of RCT by MRI and surgery were not statistically significant: the marginal homogeneity test was 0.558 , analyzing that two procedures have comparable diagnostic value.

For the diagnosis of full-thickness rotator cuff tears by MRI (Figure 2), there was a high degree of sensitivity, specificity and accuracy $(100 \%, 98.7 \%$ and $98.8 \%)$ respectively but these percentages are based on only seven cases. The size of full-thickness tears ranges from $0.7 \mathrm{~cm}$ to $4 \mathrm{~cm}$ in MRI. MRI showed only one false-positive rotator cuff tears which was proven surgically.

For the diagnosis of partial-thickness rotator cuff tears, the diagnostic values viz. sensitivity, specificity and accuracy for MRI were 87.3\%, 53.3\% and 81.3\% respectively (Table 2). MRI incorrectly identified seven partialthickness tears as false positive which were not found tears in surgery and nine partial-thickness tears as false 
Table 1. Correlation of MRI findings with Surgical findings of partial-thickness tears (PTT) and full-thickness tears (FTT).

\begin{tabular}{|c|c|c|c|c|c|}
\hline \multicolumn{6}{|c|}{ Surgical findings } \\
\hline & No Tear & Articular PTT & Bursal PTT & Full TT & Total \\
\hline \multicolumn{6}{|l|}{ MRI findings } \\
\hline No Tear & 0 & 8 & 1 & 0 & 9 \\
\hline Articular PTT & 4 & 37 & 2 & 0 & 43 \\
\hline Bursal PTT & 2 & 1 & 23 & 0 & 26 \\
\hline Full TT & 0 & 0 & 1 & 7 & 8 \\
\hline Total & 6 & 46 & 27 & 7 & 86 \\
\hline
\end{tabular}

Table 2. The diagnostic parameters of MRI for the diagnosis of partial-thickness tears (PTT) and full-thickness tears (FTT).

\begin{tabular}{ccc}
\hline & & Magnetic Resonance Imaging (MRI) \\
\hline & PTT & FTT \\
\hline Sensitivity & $62 / 71$ & $7 / 7$ \\
& {$[87.3 \%]$} & {$[100 \%]$} \\
Specificity & $8 / 15$ & $78 / 79$ \\
& {$[53.3 \%]$} & {$[98.7 \%]$} \\
PPV & $62 / 69$ & $7 / 8$ \\
& {$[89.8 \%]$} & {$[87.5 \%]$} \\
NPV & $8 / 17$ & $78 / 78$ \\
& {$[47 \%]$} & {$[100 \%]$} \\
Accuracy & $70 / 86$ & $85 / 86$ \\
& {$[81.3 \%]$} & {$[98.8 \%]$} \\
\hline
\end{tabular}

PTT: partial-thickness rotator cuff tears, FTT: full-thickness rotator cuff tears, PPV: positive predictive value, NPV: negative predictive value.

Table 3. Correlation between MRI findings and surgical findings for the diagnosis of rotator cuff tears on the basis of site as a variable.

\begin{tabular}{lcccc}
\hline & Site 2 & & \\
& No Tear & Articular PTT & Bursal PTT & Full TT \\
\hline Site1 & & & 1 & 0 \\
No Tear & 0 & 8 & 2 & 0 \\
Articular PTT & 4 & 37 & 23 & 7 \\
Bursal PTT & 2 & 1 & & 7 \\
Full TT & 0 & 0 & & \\
Kappa ${ }^{*}$ 0.645 & & & & \\
Marginal Homogeneity test : P $=0.558$ & & & \\
\hline
\end{tabular}

Site 1: types of site found in MRI Site 2: types of site found in surgery *Agreement between MRI and surgery. The standard error is given in parenthesis $\sim$ Test for significant differences between MRI and Surgery Articular PTT: articular partial-thickness rotator cuff tears, Bursal PTT: bursal partial-thickness rotator cuff tears, Full TT : full-thickness rotator cuff tears. 
negative which were found tears only in surgery (Table 4). None of them were involving intratendinously. Out of 7 false positive PTT, 5 PTT have involved articular site and 2 PTT have involved bursal site. In these false positive PTT, the signal distortion in rotator cuff muscle tendon is more suggestive of tendinosis than of partialthickness tears. Also out of 9 false negative PTT, 8 were of articular type and 1 were bursal type. On retrospective analysis of MRI findings, signal intensity of these lesions were more suggestive of PTT than of tendinopathy and that of normal intensity of rotator cuff muscle tendon.

The size of PTT ranges from $0.5 \mathrm{~cm}$ to $4 \mathrm{~cm}$ in MRI and overall mean size is $1.84 \mathrm{~cm}$.

These partial-thickness rotator cuff tears were situated superficially, at articular side $(n=43,55 \%)$ and bursal $(\mathrm{n}=26,30.2 \%)$. The full-thickness rotator cuff tears comprised only $9.3 \%(\mathrm{n}=8)$. Most of the RCT lies within 1 - $<2 \mathrm{~cm}$ range of size $(\mathrm{n}=35,40.7 \%)$. L-shape comprises highest frequency $(\mathrm{n}=32,37.2 \%)$ followed by crescentic shape $(n=27,31.4 \%)$. Supraspinatus muscle is found to be the most involved muscle $(n=54,62.8 \%)$ followed by infraspinatus $(n=15,17.4 \%)$, teres minor $(n=4,4.7 \%)$ and subscapularis $(n=4,4.7 \%)$ (Table 5).

Surgery demonstrated no rotator cuff tears and rotator cuff tears in 7 shoulders and 79 shoulders respectively (Table 6). MRI correctly depicted no tears in 0 shoulder. MRI incorrectly identified 7 shoulders as tears, which were not seen in surgery as tears (Table 6).

Table 4. Cross tabulation for diagnostic parameters of partial-thickness rotator cuff tears (PTT).

\begin{tabular}{|c|c|c|c|}
\hline & \multicolumn{3}{|c|}{ РTT2 } \\
\hline & No Tear & Tear & Total \\
\hline \multicolumn{4}{|l|}{ PTT1 } \\
\hline No Tear & 8 & 9 & 17 \\
\hline Tear & 7 & 62 & 69 \\
\hline Total & 15 & 71 & 86 \\
\hline
\end{tabular}

PTT1: partial-thickness tears found in MRI; PTT2: partial-thickness tears found in surgery; PTT: partial-thickness rotator cuff tears.

Table 5. Observed frequency in the diagnosis of PTT and FTT by MRI on the basis of site, size, shape and muscles involved as various variables.

\begin{tabular}{|c|c|c|c|c|c|}
\hline & Frequency & Percent (\%) & & Frequency & Percent (\%) \\
\hline Site & & & Shape & & \\
\hline No Tear & 9 & 10.5 & No Tear & 9 & 10.5 \\
\hline Articular PTT & 43 & 55 & Crescentic & 27 & 31.4 \\
\hline Bursal PTT & 26 & 30.2 & L-shape & 32 & 37.2 \\
\hline Full TT & 8 & 9.3 & U-shape & 18 & 20.9 \\
\hline Total & 26 & 100 & Total & 86 & 100 \\
\hline Size & & & Muscles involved & & \\
\hline No Tear & 9 & 10.5 & No Tear & 9 & 10.5 \\
\hline$<1 \mathrm{~cm}$ & 24 & 27.9 & Supraspinatus & 54 & 62.8 \\
\hline $1-<2 \mathrm{~cm}$ & 35 & 40.7 & Infraspinatus & 15 & 17.4 \\
\hline $2-<3 \mathrm{~cm}$ & 7 & 8.1 & Teres minor & 4 & 4.7 \\
\hline$\geq 3 \mathrm{~cm}$ & 11 & 12.8 & Subscapularis & 4 & 4.7 \\
\hline Total & 86 & 100 & Total & 86 & 100 \\
\hline
\end{tabular}


Table 6. Correlation of MRI findings with surgical findings of RCT overall.

\begin{tabular}{|c|c|c|c|c|c|c|}
\hline \multicolumn{7}{|c|}{ Surgery } \\
\hline & No Tears & RC Tears & Total & & Frequency & Percent (\%) \\
\hline MRI & & & & MRI & & \\
\hline No Tears & 0 & 9 & 9 & No Tears & 9 & 10.5 \\
\hline RC Tears & 7 & 70 & 77 & RC Tears & 77 & 89.5 \\
\hline Total & 7 & 79 & 86 & & & \\
\hline \multicolumn{7}{|c|}{ Sensitivity $=70 / 79=88.6 \%$} \\
\hline Accuracy $=$ & $81.3 \%$ & & & & & \\
\hline
\end{tabular}

RC Tear: Partial-thickness and full-thickness rotator cuff tears considered as tears.

On the basis of size, the consistency between MRI and surgery was poor with Kappa coefficient 0.307 . The differences in diagnosis of RCT by MRI and surgery were not significant: the marginal homogeneity test was 0.16 , suggesting the two diagnostic procedures have comparable diagnostic value.

On the basis of shape and muscles involved of RCT, the consistency between MRI and surgery was poor for shape variable with Kappa coefficient 0.358 and that for muscles involved was moderate with Kappa coefficient 0.491. Both tests have comparable diagnostic value on the basis of muscles involved as its McNemar test showed insignificant $(\mathrm{P}=0.206)$ but comparable diagnostic value was absent between them as the Mcnemar test is significant $(\mathrm{P}=0.002)$ on the basis of shape variable of RCT (Table 7).

In this study, 24 more other findings were observed in addition to RCT. Out of them 13 variables were found significant.

From the Table 8 we can conclude that humeral head edema comprised the highest sensitivity (100\%) among other findings followed by shoulder joint effusion (90.5\%). Shoulder joint hematoma had the highest specificity (100\%) followed by impingement syndrome (97.3\%). The agreement between MRI and surgery in diagnosing calcific tendinitis and shoulder joint hematoma in addition to RCT with Kappa coefficient (0.577) and (0.556) respectively were moderate and better than that for other findings although the differences in diagnosing them was insignificant with McNemar test ( $\mathrm{P}>0.05$ for both), which showed that there is comparable diagnostic value between MRI and surgery. The McNemar test for shoulder joint effusion, biceps tendon effusion, biceps tendon injury, subacromial bursitis, humeral head edema, humeral head cyst, synovial hyperplasia were found to be significant $(\mathrm{P}<0.05)$ although the agreement between MRI and surgery for diagnosing them were poor with Kappa coefficient $<0.4$ for each.

\section{Discussion}

In this study, we compared the accuracy of MRI in the detection of rotator cuff tears in patients who underwent surgical procedures as therapeutic purposes. In our hospital, MRI is the investigation of choice in patients with chronic shoulder joint complaints. Arthrography, an invasive technique, has been used for the diagnosis of RCT which has well-recognized risks [17] [18]. So the use of MRI has increased tremendously in most of the countries for imaging shoulder as a diagnostic tool [19]. In our study, the use of MRI with updated imaging technology has shown a promising diagnostic value for the pathology of shoulder joint.

The reproducibility and moderate reliability of history and clinical diagnosis [7] and the high life time prevalence of shoulder pain of 66\% [20] may be an answer for more frequently use of MRI examinations. In this research, we have mainly focused on absence and presence of rotator cuff tears on the basis of different variables which includes size, shape, site and muscles involved of RCT. Other findings in addition to RCT have been also mentioned; which were not evaluated in Matthieu et al.; combination of RCT and associated other pathology in MRI examination is found to have significant diagnostic value. Beside it, clinical history and good clinical examination can fulfill the need for the diagnostic certainty in RCT and the surgical approach for it carries better therapeutic value. 
Table 7. Evaluation for the diagnosis of RCT on the basis of various variables.

\begin{tabular}{ccc}
\hline & Kappa coefficient & McNemar Bowker Test \\
\hline Size & Value & Asymp. Sig (P) \\
Shape & 0.307 & 0.16 \\
Muscles involved & 0.358 & 0.002 \\
\hline
\end{tabular}

Table 8. Correlation and the agreement of MRI and surgery in the diagnosis of other significant findings in addition to RCT.

\begin{tabular}{|c|c|c|c|c|c|}
\hline & Sensitivity (\%) & Specificity (\%) & Frequency (MRI) & Kappa coefficient & $\begin{array}{l}\text { McNemar Test } \\
\text { (Approx.Sig.) }\end{array}$ \\
\hline SJE & 90.5 & 23.3 & $65(75.6 \%)$ & 0.082 & 0.000 \\
\hline BTE & 89.5 & 46.8 & $50(58.1 \%)$ & 0.231 & 0.000 \\
\hline BTI & 12.5 & 96.5 & $5(5.8 \%)$ & 0.117 & 0.000 \\
\hline $\mathrm{SAB}$ & 16.7 & 87.7 & $11(12.8 \%)$ & 0.052 & 0.019 \\
\hline $\mathrm{HF}$ & 60.0 & 85.9 & $16(18.6 \%)$ & 0.365 & 0.180 \\
\hline HHE & 100 & 75.6 & $22(25.6 \%)$ & 0.187 & 0.000 \\
\hline HHC & 0.00 & 82.3 & $14(16.3 \%)$ & 0.045 & 0.004 \\
\hline SJH & 40.0 & 100 & $2(2.3 \%)$ & 0.556 & 0.250 \\
\hline SHyp & 4.1 & 93.8 & $4(4.7 \%)$ & 0.017 & 0.000 \\
\hline CT & 53.3 & 97.0 & $10(11.6 \%)$ & 0.577 & 0.180 \\
\hline IS & 28.6 & 97.3 & $4(4.7 \%)$ & 0.321 & 0.453 \\
\hline ACA & 33.3 & 96.0 & $5(5.8 \%)$ & 0.318 & 1.000 \\
\hline SJS & 50.0 & 94.8 & $6(7 \%)$ & 0.362 & 0.688 \\
\hline
\end{tabular}

SJE: shoulder joint effusion, BTE: biceps tendon effusion, BTI: biceps tendon injury, SAB: subacromial bursitis, HF: humerus fracture, HHE: humeral head edema, HHC: humeral head cyst, SJH: shoulder joint hematoma, SHyp: synovial hyperplasia, CT: calcific tendinitis, IS: impingement syndrome, ACA: acromioclavicular arthritis, SJS: shoulder joint subluxation.

As the indication to perform MRI of shoulder was not only of suspicion for having rotator cuff tears, MRI had found a large number of negative findings. Another disadvantage of MRI examination could be a large number of false positive results, which might be as per similarity of signal intensity of different pathological conditions of shoulder due to the technical aspects and the chronicity of the lesions. Milgrom C et al. mentioned that there is a chance of up to $50 \%$ of findings abnormalities in an asymptomatic shoulder [21].

Evaluation of the diagnostic accuracy of MRI in RCT was another purpose of this retrospective study. For this by comparing with the surgical findings of RCT, the diagnostic accuracy (percentage of correct diagnosis) of MRI have been calculated which showed $81.3 \%$ and $98.8 \%$ for PTT and FTT respectively. Although for FTT, its diagnostic accuracy is excellent, it was less but comparably accurate for the detection of PTT. Our results substantiate these reported by Matthieu et al. [7], Teefey et al. [11] and Gohar Abbas Naqvi et al. [22], who showed that MRI have comparable accuracy for depicting PTT and FTT. Matthieu et.al performed the study retrospectively with larger number of patient population $(\mathrm{n}=275)$ and also included large number of true negative patient and followed ultrasound examination as well. Teefey et al. [11] performed a prospective study to show the diagnostic accuracy of MRI in diagnosing RCT.

In our study, we have included more nominal data as different variables for RCT to find MRI accuracy which were absent in previous study. Gohar Abbas Naqvi et al. mentioned 91 cases who had evaluated accuracy of MRI in detecting RCT retrospectively with only one nominal data as a variable i.e. presence of tears or absence of tears of supraspinatus tendon only and also categorized only to FTT in different types as per the length of tear 
[22]. Matthieu et al. had evaluated diagnostic accuracy with only two variables. In our study almost everyone had undergone MRI 3.0T examination so we had additional better diagnostic value to assess other associated findings like fatty infiltration, impingement syndrome, labrum tear, glenohumeral ligament injury etc. and also to define the precise location, extent, shape and rotator cuff muscles involvement which were absent in Matthieu et al. [7].

Our study has some limitations. The operator dependency of MRI is a potential drawback [14] [23]. There still exists possibility of variability in the performances interpreting the scan between radiologists, as well as between the radiologists and surgeons [24]. We have compared all the types of variables of MRI with that of surgery. If there was no technical drawback, the interobserver agreement is good. The Kappa coefficient was calculated to be 0.645 , which was for site variable of RCT.

As the study was not done with random selection of patient and did not include the true negative cases, it was prone to bias. For example the study of patients of 804 cases from Jan 2012 to Sep 2015 who underwent MRI for shoulder but not surgery certainly differs from who were operated upon (selection bias). Variation caused by sample differences can cause wide variation in results.

As the imaging results were preoperatively known by orthopedic surgeons, the decision making to treat surgically or not had heavily influenced the patient selection (verification or workup bias) [7] which lead to diagnostic review bias influencing the gold standard. Thus the reference standard, the so-called gold standard possesses an imperfect standard bias [7].

Waldt et al. [25] showed that at muscle tendon margin differentiation among fibre tearing, tendinitis, tendinopathy, synovial changes and superficial fraying is difficult so the diagnosis of small PTT are restricted. There occurs limitation to find out obvious focal discontinuity or defect depending on the presence of high signal intensity only which lead to underestimation of true PTT [26]. We experienced reference standard was more dependent upon MRI findings, especially while detecting PTT (Figure 3). Few false negatives were read as either reactive changes due to tendinopathy, tendinosis or calcific tendinitis and still it is not uncommon even using high-field MRIs [24]. Using high-field MRI scanners, improvement in identifying the shoulder pathology has been shown by arthrograms [24]. In our study, none of the cases had been undergone arthrogram. There were seven false positive PTT and if these PTT were regarded as true positive then the sensitivity, specificity, accuracy, positive predictive value, negative predictive value of MRI would increase as that of FTT. Thus the imperfect standard bias and selection bias has hugely decreased the accuracy of MRI in detection of PTT.

If diagnostic radiologists ignore increasing requests of MRI examination for better evaluation in diagnosis of RCT, these MRI slides will soon be reported by orthopedic surgeons and physicians by themselves [27]-[29].

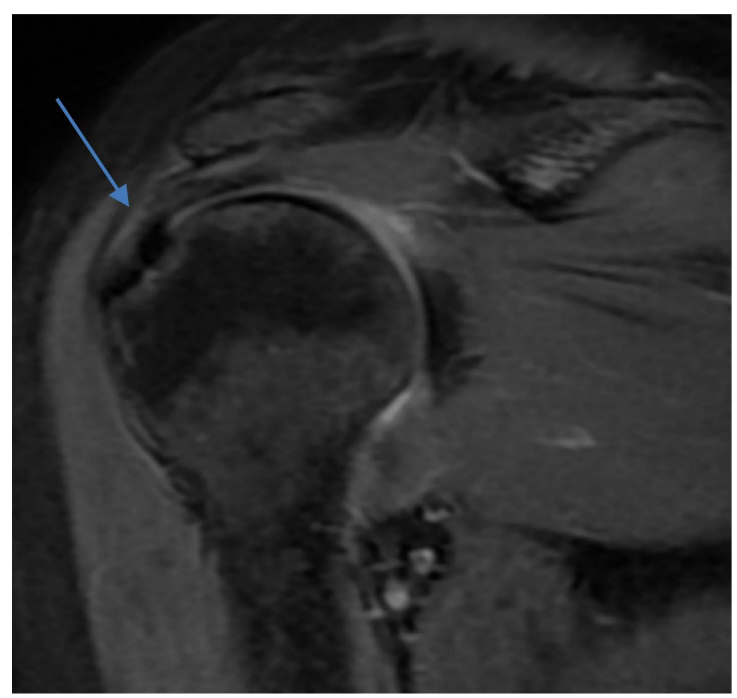

Figure 3. Coronal T2 weighted fat-suppression MR image show a partialthickness tears (arrow) at the insertion of supraspinatus tendon which were wrongly interpreted as tears for thickened tendon (false-positive partialthickness rotator cuff tears). This finding was confirmed as no tears in surgery. Also shoulder joint effusion can be seen. 


\section{Conclusion}

For patients with acute and chronic shoulder discomfort, MRI is very reliable imaging investigation for both PTT and FTT despite its relatively high cost and occasional limited availability. The super high-field scanners provide superior imaging quality, but it is not without limitations. To detect PTT, the ability of MRI is less well established than that for detecting FTT. It has high accuracy, sensitivity and positive predictive value in diagnosing FTT. Also although it has less accuracy for diagnosing PTT but has sensitivity and positive predictive value as good as that for diagnosing FTT. As there is high sensitivity, specificity and the consistency is found to be moderate in diagnosing RCT between MRI and surgery on the basis of site and muscle involved and differences in diagnosing RCT between them for some of associated other findings in addition to RCT are significant, combining them in the MRI findings offers a great value in the detection of RCT. Of course dedicated expertise and his/her preference, local setting, equipment availability and its strength, patient preference and cost effectiveness do play a vital role in preferring the imaging technique to be selected as a diagnostic investigation.

\section{Acknowledgements}

The authors thank the following radiologists for reading the images in this study: Ju Sheng Hong, Ph.D.; Jing Ya Ling, Ph.D.; Rajendra Darai, Ph.D. Beena Darai, M.N., Rudra Darai, M.A., M.S., Gyanu Darai, PCL, B.A., Saroj Shah, MBBS and all the staffs of Department of Radiology, Southeast University, Nanjing, China.

\section{References}

[1] Mitchell, C., Adebajo, A., Hay, E. and Carr, A. (2005) Shoulder Pain: Diagnosis and Management in Primary Care. BMJ, 331, 1124-1128. http://dx.doi.org/10.1136/bmj.331.7525.1124

[2] Macfarlane, G.J., Hunt, I.M. and Silman, A.J. (1998) Predictors of Chronic Shoulder Pain: A Population Based Prospective Study. The Journal of Rheumatology, 25, 1612-1615.

[3] Dalton, S.E. (1994) The Conservative Management of Rotator Cuff Disorders. Rheumatology, 33, 663-667. http://dx.doi.org/10.1093/rheumatology/33.7.663

[4] Seibold, C.J., Mallisee, T.A., Erickson, S.J., Boynton, M.D., Raasch, W.G. and Timins, M.E. (1999) Rotator Cuff: Evaluation with US and MR Imaging. RadioGraphics, 19, 685-705. http://dx.doi.org/10.1148/radiographics.19.3.g99ma03685

[5] Iannotti, J.P., Zlatkin, M.B., Esterhai, J.L., Kressel, H.Y., Dalinka, M.K. and Spindler, K.P. (1991) Magnetic Resonance Imaging of the Shoulder. The Journal of Bone \& Joint Surgery, American Volume, 73, 17-29.

[6] Singson, R.D., Hoang, T., Dan, S. and Friedman, M. (1996) MR Evaluation of Rotator Cuff Pathology Using T2Weighted Fast Spin-Echo Technique with and without Fat Suppression. American Journal of Roentgenology, 166, 1061-1065. http://dx.doi.org/10.2214/ajr.166.5.8615243

[7] Rutten, M.J.C.M., Spaargaren, G.-J., van Loon, T., de Waal Malefijt, M.C., Kiemency, L.A.L.M. and Jager, G.J. (2010) Detection of Rotator Cuff Tears: The Value of MRI Following Ultrasound. European Radiology, 20, 450-457. http://dx.doi.org/10.1007/s00330-009-1561-9

[8] Chang, C.Y., Wang, S.F., Chiou, H.J., Ma, H.L., Sun, Y.C. and Wu, H.D. (2002) Comparison of Shoulder Ultrasound and MR Imaging in Diagnosing Full-Thickness Rotator Cuff Tears. Clinical Imaging, 26, 50-54. http://dx.doi.org/10.1016/S0899-7071(01)00323-0

[9] Swen, W.A., Jacobs, J.W., Algra, P.R., et al. (1999) Sonography and Magnetic Resonance Imaging Equivalent for the Assessment of Full-Thickness Rotator Cuff Tears. Arthritis \& Rheumatism, 42, 2231-2238. http://dx.doi.org/10.1002/1529-0131(199910)42:10<2231::AID-ANR27>3.0.CO;2-Z

[10] Martin-Hervas, C., Romero, J., Navas-Acien, A., Reboiras, J.J. and Munuera, L. (2001) Ultrasonographic and Magnetic Resonance Images of Rotator Cuff Lesions Compared with Arthroscopy or Open Surgery. Journal of Shoulder and Elbow Surgery, 10, 410-415. http://dx.doi.org/10.1067/mse.2001.116515

[11] Teefey, S.A., Rubin, D.A., Middleton, W.D., Hildebolt, C.F., Leibold, R.A. and Yamaguchi, K. (2004) Detection and Quantification of Rotator Cuff Tears. Comparison of Ultrasonographic, Magnetic Resonance Imaging, and Arthroscopic Findings in Seventy-One Consecutive Cases. The Journal of Bone \& Joint Surgery, American Volume, 86-A, 708-716.

[12] Fotiadou, A., Vlychou, M., Papadopoulos, P., Karataglis, D.S., Palladas, P. and Fezoulidis, I.V. (2008) Ultrasonography of Symptomatic Rotator Cuff Tears Compared with MR Imaging and Surgery. European Journal of Radiology, 68, 174-179. http://dx.doi.org/10.1016/j.ejrad.2007.11.002 
[13] Dinnes, J., Loveman, E., McIntyre, L. and Waugh, N. (2003) The Effectiveness of Diagnostic Tests for the Assessment of Shoulder Pain Due to Soft Tissue Disorders: A Systematic Review. Health Technology Assessment, 7, 1-166. http://dx.doi.org/10.3310/hta7290

[14] Balich, S.M., Sheley, R.C., Brown, T.R., Sauser, D.D. and Quinn, S.F. (1997) MR Imaging of the Rotator Cuff Tendon: Interobserver Agreement and Analysis of Interpretive Errors. Radiology, 204, 191-194. http://dx.doi.org/10.1148/radiology.204.1.9205245

[15] Jbara, M., Chen, Q., Marten, P., Morcos, M. and Beltran, J. (2005) Shoulder MR Arthrography: How, Why, When. Radiologic Clinics North America, 43, 683-692. http://dx.doi.org/10.1016/j.rcl.2005.01.004

[16] Kassarjian, A., Bencardino, J.T. and Palmer, W.E. (2006) MR Imaging of the Rotator Cuff. Radiologic Clinics of North America, 44, 503-523. http://dx.doi.org/10.1016/j.rcl.2006.04.005

[17] Mink, J.H., Harris, E. and Rappaport, M. (1985) Rotator Cuff Tears: Evaluation Using Double-Contrast Shoulder Arthrography. Radiology, 157, 621-623. http://dx.doi.org/10.1148/radiology.157.3.4059549

[18] Goldman, A.B. and Ghelman, B. (1978) The Double-Contrast Shoulder Arthrogram: A Review of 158 Studies. Radiology, 127, 655-663. http://dx.doi.org/10.1148/127.3.655

[19] Awerbuch, M.S. (2008) The Clinical Utility of Ultrasonography for Rotator Cuff Disease, Shoulder Impingement Syndrome and Subacromial Bursitis. The Medical Journal of Australia, 188, 50-53.

[20] Luime, J.J., Koes, B.W., Hendriksen, I.J., et al. (2004) Prevalence and Incidence of Shoulder Pain in the General Population; A Systematic Review. Scandinavian Journal of Rheumatology, 33, 73-81. http://dx.doi.org/10.1080/03009740310004667

[21] Milgrom, C., Schaffler, M., Gilbert, S. and van Holsbeeck, M. (1995) Rotator Cuff Changes in Asymptomatic Adults: The Effect of Age, Hand Dominance, and Gender. The Journal of Bone \& Joint Surgery, 77, 296-298.

[22] Naqvi, G.A., Jadaan, M. and Harrington, P. (2009) Accuracy of Ultrasonography and Magnetic Resonance Imaging for Detection of Full Thickness Rotator Cuff Tears. International Journal of Shoulder Surgery, 3, 94-97. http://dx.doi.org/10.4103/0973-6042.63218

[23] Robertson, P.L., Schweitzer, M.E., Mitchell, D.G., et al. (1995) Rotator Cuff Disorders: Interobserver and Intraobserver Variation in Diagnosis with MR Imaging. Radiology, 194, 831-835. http://dx.doi.org/10.1148/radiology.194.3.7862988

[24] Lee, C.S., Davis, S.M., McGroder, C., Kouk, S., Sung, R.M., Stetson, W.B. and Powell, S.E. (2014) Analysis of Low-Field MRI Scanners for Evaluation of Shoulder Pathology Based on Arthroscopy. Orthopaedic Journal of Sports Medicine, 2, Article ID: 2325967114540407.

[25] Waldt, S., Bruegel, M., Mueller, D., et al. (2007) Rotator Cuff Tears: Assessment with MR Arthrography in 275 Patients with Arthroscopic Correlation. European Radiology, 17, 491-498. http://dx.doi.org/10.1007/s00330-006-0370-7

[26] Escobedo, E.M., Hunter, J.C., Hollister, M.C., Pattern, R.M. and Goldstein, B. (1997) MR Imaging of Rotator Cuff Tears in Individuals with Paraplegia. American Journal of Roentgenology, 168, 919-923.

[27] Iannotti, J.P. and Kwon, Y.W. (2005) Management of Persistent Shoulder Pain: A Treatment Algorithm. The American Journal of Orthopedics, 34, 16-23.

[28] Roberts, C.S., Galloway, K.P., Honaker, J.T., Hulse, G. and Seligson, D. (1998) Sonography for the Office Screening of Suspected Rotator Cuff Tears: Early Experience of the Orthopedic Surgeon. The American Journal of Orthopedics, 27, 503-506.

[29] Al-Shawi, A., Badge, R. and Bunker, T. (2008) The Detection of Full Thickness Rotator Cuff Tears Using Ultrasound. The Bone \& Joint Journal, 90, 889-892. http://dx.doi.org/10.1302/0301-620X.90B7.20481 